\title{
The Role of Right Interpretation of Space Charge Distribution for Optimized Design of HVDC Cables
}

\author{
A. Imburgia, P. Romano, Senior Member, IEEE, G. Ala, Senior Member, IEEE, E. Riva Sanseverino, \\ G. Giglia
}

\begin{abstract}
In the field of high voltage transmission systems, different degradation phenomena affect the reliability of the employed components. In particular, under DC stress, the space charge accumulation phenomenon is believed the most responsible of the dielectrics lifetime reduction. To measure the accumulated space charges in flat specimens, the PEA method is one of the most used techniques. The working principle of the PEA cell is based on the acoustic waves propagation and detection. As is well known, the acoustic waves propagating in different means are partially transmitted and partially reflected. Therefore, the piezoelectric sensor of the PEA cell is subjected of both direct and reflected waves. The latter, which are also present in the PEA cell output signal, if are not taken into account could be easily confused as a charge accumulation. For this reason, in this work the acoustic waves behavior within the PEA cell has been simulated in order to avoid incorrect interpretation of the PEA cell output charge profiles. In this paper, compared to other works present in literature in which only the surface charges were considered, the space charges accumulated in the insulation bulk are also considered for a complete analysis of the waves behavior during the PEA measures. The results found in the present work are very useful for cables manufacturing industries in which the choice of the dielectric materials is strongly influenced by the space charge accumulation phenomenon.
\end{abstract}

Index Terms - Space charge, PEA method, HVDC, modelling, FDTD method.

\section{INTRODUCTION}

$\mathrm{I}_{\mathrm{p}}^{\mathrm{N}}$ $\mathrm{N}$ high voltage systems, especially under AC stress, the partial discharges phenomena are considered the main dielectric degradation causes [1-5]. In high voltage direct current (HVDC) systems, instead, the space charge

This paragraph of the first footnote will contain the date on which you submitted your paper for review. It will also contain support information, including sponsor and financial support acknowledgment. For example, "This work was supported in part by the U.S. Department of Commerce under Grant BS123456."

The next few paragraphs should contain the authors' current affiliations, including current address and e-mail. For example, F. A. Author is with the National Institute of Standards and Technology, Boulder, CO 80305 USA (email: author@boulder.nist.gov).

S. B. Author, Jr., was with Rice University, Houston, TX 77005 USA. He is now with the Department of Physics, Colorado State University, Fort Collins, CO 80523 USA (e-mail: author@lamar.colostate.edu).

T. C. Author is with the Electrical Engineering Department, University of Colorado, Boulder, CO 80309 USA, on leave from the National Research Institute for Metals, Tsukuba, Japan (e-mail: author@nrim.go.jp). accumulation phenomenon is believed the main factor causing the dielectric lifetime reduction [6-8]. Considering that, several space charge measurement techniques were proposed [9]. By making a comparison among the all techniques, the Pulsed Electro-Acoustic PEA method [10] can be considered the most simply to be used [11]. This measurement method is essentially founded on the acoustic waves originated by charges vibration and on its detection, after propagating within the PEA cell, by means of a piezoelectric sensor. Considering the PEA cell is composed by different components with different materials, the reflection phenomenon occurs [12]. Therefore, based on the PEA cell components and sample features, the PEA cell output profiles could be affected by noise signals due to wave reflections. This, means that the obtained charge distribution is distorted and thus the real charges accumulation distributions could be misinterpreted.

In order to analyze and better understand the acoustic wave behavior, in literature several PEA cell models are reported.

In [13], Galloy et al elaborated a one-dimensional electroacoustic model based on the Redwood's equivalent circuit of piezoelectric and non-piezoelectric materials for the acoustic part of a PEA cell. In particular, they found a way to improve the charge distribution resolution by optimizing the values of the acoustic detector parameters.

Another PEA cell simulation model has been developed with commercial software COMSOL ${ }^{\circledR}$ [14]. In this work, the effect of the piezoelectric sensor thickness in the PEA cell output signal was evaluated, without considering the attenuation and dispersion of acoustic waves.

In [15], Chahal and Reddy, proposed a PSpice model in order to simulate the acoustic waves behavior within the PEA cell. The authors modeled the entire PEA cell by means of cascade connected lossy transmission lines. In this work, the effect of the PEA cell components dimensions in the output profile was analyzed.

In the articles above mentioned, as well as in that previous published by the authors [16], only the surface charges deposited in the sample interfaces were taken into account. In the proposed work, instead, the developed model has been implemented in Matlab environment and for a complete analysis of the waves behavior during the PEA measures, the space charges accumulated in the insulation bulk are also considered. Thanks to the use of numerical software, especially in the case of accumulated space charges, the implementation of the pulse generators employed to simulate 
the acoustic wave generation, becomes simple and fast.

For a complete and understandable explanation of the proposed work, in Section 2 a brief description of the PEA method is given. While, in Section 3, the schemes representing the acoustic wave behavior and the related description, are reported in detail.

After that, the modeling approach and how it was set up, are described. In the last sections, instead, the simulations and the model validation are shown.

Concerning the simulations. Initially, the case in which the sample features provide a clear PEA output signal (without reflections within it), is described. After that, simulation showing the output signal distortion, due to the reflections of acoustic wave within the ground electrode and absorber, are also reported. In order to theoretically validate the developed model, the obtained simulation results have been compared with those found in literature [15]. After that, the experimentally model validation has been also made by comparing simulation and experimental results. Experimental measures were made by using two different PEA cell. The first one is that used at the Tony Davis High Voltage Laboratory (TDHVL) of the University of Southampton. The second one is the PEA cell of the LEPRE high voltage lab located at the University of Palermo. This validation has been performed for an output signal without and with distortion, and the case of undistorted output signal with the presence of space charges.

\section{THE PEA METHOD}

In Figure 1, a schematic representation of the PEA cell is reported. Its working principle can be explained as follow.

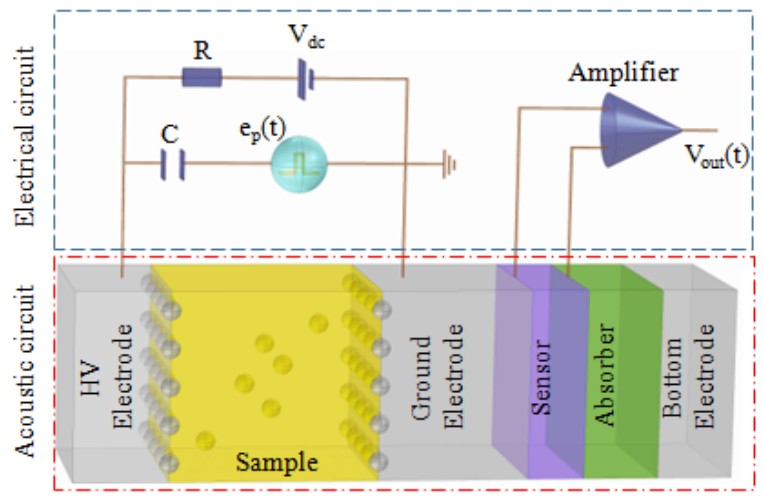

Fig. 1. Working principle of the PEA method. The electrical and acoustic circuits are highlighted by the blue and red dashed boxes, respectively.

Initially, the high voltage $\mathrm{V}_{\mathrm{dc}}$ generator is applied in order to create a constant DC stress in the sample and promote the space charges generation and accumulation. Then, the accumulated charges are subjected to a vibration by means of the pulse generator $e_{p}(t)$. This vibration generates acoustic waves that after travelling within the PEA cell are detected by the piezoelectric sensor in which are converted to a voltage signal. The component in green color is a waves absorber, it is made by the same material of the piezoelectric sensor (polyvinylidene fluoride, PVDF), and thus it avoids reflections after the acoustic waves pass the sensor. The electrode on the right side, named bottom electrode, which it is not present in all PEA cell, is used for the mechanical protection of the electronic components place inside the cell.

As can be seen in Figure 1, the PEA cell has been divided in two blocks, the electrical and the acoustic part. The electrical part is composed by electrical components, such as resistance $\mathrm{R}$, capacitance $\mathrm{C}$, amplifier and the two generators. The acoustic part, instead, is constituted by the ground, the bottom and the high voltage (HV) electrodes. But also by the other components in which the acoustic waves are travelling, such as the sample, the piezoelectric sensor and the absorber [8].

Acoustic waves behavior within a pea cell

One of the main issues occurring during space charge measurement with the PEA method is the correct evaluation of the cell output signal. The PEA cell output charge profile, for a single layer specimen and space charges free, is composed by only two peaks related to the sample interfacial (see Figure 4 of [17]). In this case the positive and negative peaks are separated at a distance depending on sample thickness and sound velocity. In the ideal case, without wave reflections, no other signals should be present between the two peaks. Typically, due to the wave reflections, false signals could be present between the two main peaks and thus the PEA output signal interpretation becomes not easy.

However, the proposed model is useful to better understand the influence of some PEA cell component and of the sample under test on the acoustic wave behavior. In particular, research results show that the presence of reflections in the main output signal mainly depends on the sample features. In order to avoid this phenomenon, in this section, the relationships that should be taken into account, are reported and described.

As is well known, the reflections of acoustic waves occur in correspondence of the interfaces between two difference materials. Therefore, with reference to our case, this phenomenon is present in each PEA cell component interface a part that between the piezoelectric sensor and absorber. In Figure 2, the acoustic wave behavior within the ground electrode of the PEA cell is depicted. In the figure, $A$ is the material constituting the sample, while $P V D F$ and $A L$ (aluminum) and the materials used for the piezoelectric sensor and the electrodes, respectively.

The acoustic waves generated in correspondence of the HVelectrode/sample interface, named $p_{+}(t)$, reach the piezoelectric sensor after a time $\tau_{S a}+\tau_{G R}(\tau$ is the transit time of the acoustic wave in each PEA cell component and sample, calculated as its thickness $d$ over the related material sound velocity v).

When the wave $p_{+}(t)$ across the sample/ground-electrode interface, it is partially transmitted and partially reflected. This latter portion of wave, $p_{+}^{R-A}(t)$, based on the reflection coefficient $K_{A-A L}^{R}$, is reflected and goes back in the HVelectrode direction (the reflection $K_{i-j}^{R}$ and the transmission coefficients $K_{i-j}^{T}$, are widely explained in [18-19]).

Others reflections of $p_{+}^{R-A}(t)$ are negligible because the time 
needed to reach the piezoelectric sensor will be greater to the time taken by the original signal $p_{+}(t)$. The transmitted fraction of the wave $p_{+}(t)$ reaches the piezoelectric sensor after $\tau_{S a}+\tau_{G R}$. Simultaneously, a fraction of $p_{+}(t)$, named $p_{+}^{R_{G}}(t)$, is two times reflected in the ground electrode, according to the reflection coefficients of the involved interfaces $K_{A L-P V D F}^{R}$ and $K_{A L-A}^{R}$. After that, the reflected wave $p_{+}^{R_{G}}(t)$, is detected by the piezoelectric sensor at a time equal to $\tau_{S a}+3 \tau_{G R}$.

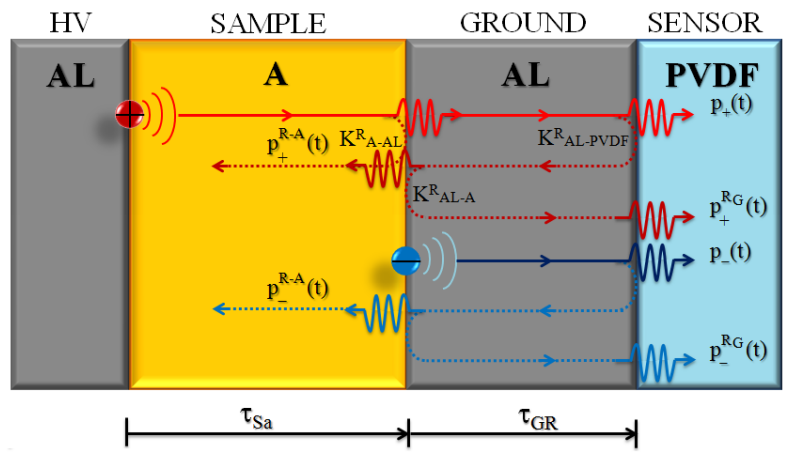

Fig. 2. Reflection of acoustic waves within the ground electrode in a single layer specimen with the presence of only interfacial charges. The acoustic waves are indicated with $\mathrm{p}$, while $\mathrm{K}^{\mathrm{R}}$ are the reflection coefficients of the different interfaces.

Regarding the waves originated in the sample/groundelectrode interface, named $p_{-}(t)$, a time $\tau_{G R}$ is necessary to be detected by the transducer. After this time, a wave portion named $p_{-}^{R_{G}}(t)$ is reflected in the ground electrode/piezoelectric sensor interface. This wave has a behavior as the wave $p_{+}^{R_{G}}(t)$. As a final point, $p_{-}^{R_{G}}(t)$, after $3 \tau_{G R}$ is sensed again by the piezoelectric sensor. While, as in the previous case of $p_{+}^{R-A}(t)$, the transmitted wave portion travelling in the $\mathrm{HV}$-electrode direction, can be neglected.

Therefore, $p_{-}^{R_{G}}(t)$ reaches the piezoelectric sensor before than $p_{+}^{R_{G}}(t)$, for this reason particular attention should be given to the wave $p_{-}^{R_{G}}(t)$ in order to evaluate if this reflected wave reaches the piezoelectric sensor for a time less than that needed to the original wave $p_{+}(t)$. For this aim, for a specified dielectric material A (with speed of sound $v_{A}$ ), based on the ground electrode dimension, the maximum sample thickness value $d_{S a}$ should be chosen in accordance with the equation 1 .

$$
d_{S a}<2 \frac{d_{G R}}{v_{A L}} v_{A}
$$

Likewise, but considering the acoustic wave behavior in the absorber component, the waves paths are depicted in Figure 3. As previously explained, the acoustic wave that reaches the piezoelectric sensor/absorber interface is totally transmitted, due to the same material used for both components. On the other hand, in the absorber/bottom-electrode interface, the related reflection coefficient $K_{P V D F-A L}^{R}$ is not zero, therefore the waves reaching this point are in part transmitted and in part reflected. Considering that the reflected waves in this last interface are travelling in the piezoelectric sensor direction, the original signal could appear distorted.

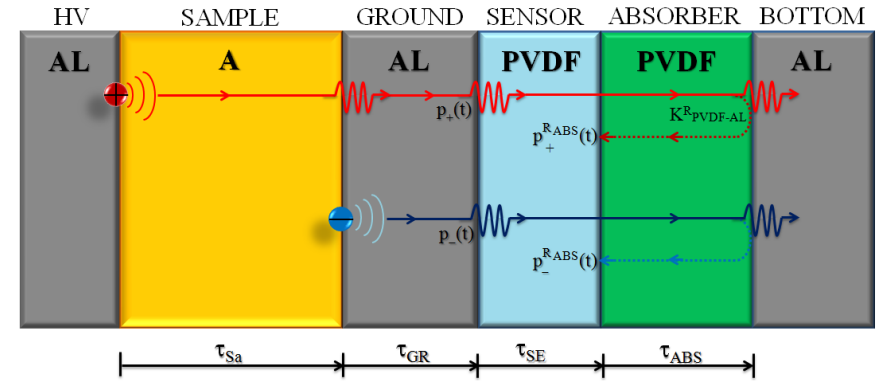

Fig. 3. Reflection of acoustic waves within the Absorber in a single layer specimen with the presence of only interfacial charges. The acoustic waves are indicated with $\mathrm{p}$, while $\mathrm{K}^{\mathrm{R}}$ are the reflection coefficients of the different interfaces.

The wave $p_{+}(t)$, needs a time given by $\tau_{S a}+\tau_{G R}$ (see Figure 2) to be detected by the piezoelectric transducer. While its reflection within the absorber $p_{+}^{R_{A B S}}(t)$ reaches the sensor after $\tau_{+}^{R_{A B S}}=\tau_{S a}+\tau_{G R}+\tau_{S E}+2 \tau_{A B S}\left(\tau_{S E}\right.$ and $\tau_{A B S}$ are the time needed for the waves to across the piezoelectric sensor and absorber, respectively). For the same aim, $p_{-}(t)$ and its reflection $p_{-}^{R_{A B S}}(t)$ need a time intervals given by $\tau_{G R}$ and $\tau_{G R}+\tau_{S E}+2 \tau_{A B S}$, respectively.

As it is possible to notice, the waves $p_{+}(t)$ and $p_{-}(t)$ reach the transducer always before $p_{+}^{R_{A B S}}(t)$, thus the latter wave does not influence the main output signal. On the contrary, the reflected wave $p_{-}^{R_{A B S}}(t)$ could anticipate $p_{+}(t)$ and then generate signal distortion if the sample thickness is greater than that provided by the equation 2 .

$$
d_{A B S}>\frac{1}{2} d_{S a} \frac{v_{P V D F}}{v_{A}}
$$

Where $v_{P V D F}$ and $d_{A B S}$ are the speed of sound and the thickness of the absorber layer, respectively.

Based on the above described, if both equations (1) and (2) are satisfied, the main PEA cell output signal is not influenced by waves reflections, also in the case in which space charges are accumulated in the insulation bulk.

In the following paragraphs, the theoretical treatment above reported is validated by simulation results.

\section{MODELING APPROACH IV-I. TWO-PORT NETWORK MODEL OF THE TRANSMISSION LINE}

When a specimen is placed between the PEA cell electrodes and it is subjected to the pulse voltage, the charges vibrations generate acoustic waves in both directions, such as the forward direction (toward the ground electrode) and the backward direction (toward the HV electrode). The acoustic waves that travel in backward directions after being reflected are also sensed by the piezoelectric sensor. Therefore, compared to the work [14], in our model all the acoustic waves are taken into account. In Figure 4, the complete acoustic wave behavior is reported. Where, the black color is used for the waves sensed by sensor after their reflection, while the red and black colors are used for the direct waves related to the positive and negative surface charges, 
respectively.

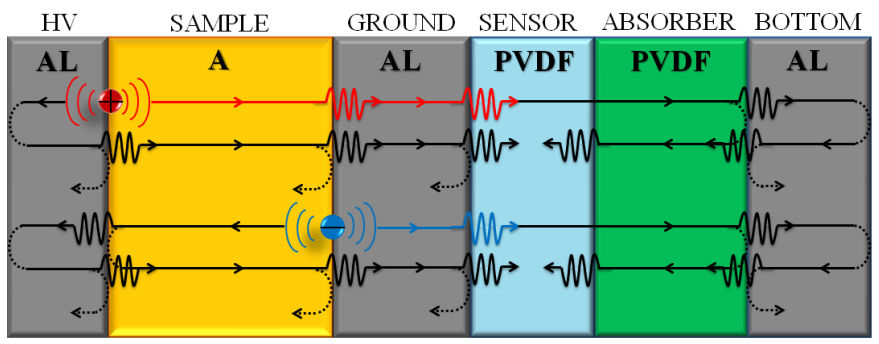

Fig. 4. Propagation of acoustic wave within the PEA cell and the specimen under test. The red and blue waves, due to positive and negative charges, propagate directly in the sensor direction. While, the black waves are sensed by the piezoelectric sensor after being reflected.

According to the analogies between mechanical and electrical quantities, such as force-voltage and velocitycurrent, it is possible to simulate an acoustic wave (described by it force and velocity) as an electrical wave (described by its voltage and current) [20]. Based on these analogies, it is also possible to simulate each mechanical PEA cell component as a lossy transmission line discretized with cascade connected two-ports, as reported in Figure 5.

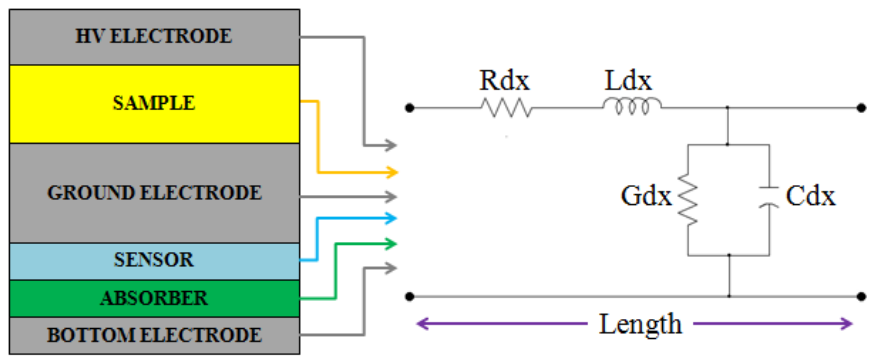

Fig. 5. Elementary two-port adopted to discretize each PEA cell component.

In particular, the transmission line parameters, such as resistance $(R)$ inductance $(L)$ capacitance $(C)$ and conductance $(G)$, can be calculated by means of the following equations:

$$
R=2 \rho v A \alpha ; \quad L=A \rho ; \quad C=\frac{1}{A \rho v^{2}} ; \quad G=\frac{2}{\rho v A} \alpha_{t c} ;
$$

where, $\rho$ is the material density, $v$ is the material sound velocity, $\alpha$ is the attenuation coefficient due to viscous losses and $\alpha_{t c}$ the attenuation coefficient due to thermal conductance. While $B$ is the cross section area which in our case correspond to the area of the HV electrode.

Based on the above, into the developed model, the acoustic waves propagating in the PEA cell have been simulated as the electrical waves propagating in electrical lossy transmission line. Therefore, the Telegraphist's Equations have been implemented [21]:

$$
\begin{aligned}
& -\frac{\partial}{\partial x} v(x, t)=R \cdot i(x, t)+L \frac{\partial}{\partial t} i(x, t) \\
& -\frac{\partial}{\partial x} i(x, t)=G \cdot v(x, t)+C \frac{\partial}{\partial t} v(x, t)
\end{aligned}
$$

where $R, L, C$ and $G$ are given by the previously reported equations (3), while $v(x, t)$ and $i(x, t)$ are the voltage and current waves, respectively.

\section{VI-II. THE NUMERICAL IMPLEMENTATION OF TELEGRAPHIST'S EQUATIONS}

In this work, to calculate and simulate the Telegraphist's equations, and therefore to visualize the propagation of voltage and current in transmission line (or pressure and velocity within PEA cell), the finite-difference time-domain (FDTD) method has been used [22-23]. This is a technique in which the derivatives with respect to space $x$ and time $t$ are approximated by finite differences applied in a spatial and temporal lattice, as:

$$
\frac{\partial}{\partial t} \cong \frac{\Delta}{\Delta t} ; \quad \quad \frac{\partial}{\partial x} \cong \frac{\Delta}{\Delta x} ;
$$

Where $\Delta t$ and $\Delta x$ are the time step and the space step, respectively.

Initially, in the application of the FDTD algorithm, a lattice is defined as a set of discrete points in space and time that samples the functions [22]. This lattice is shown in Figure 6, in which the distances between two points in space, $\Delta x$, and in time, $\Delta t$, are fixed.

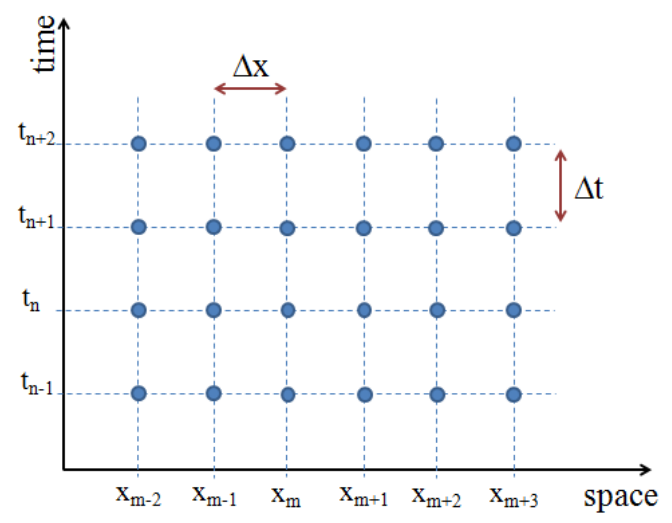

Fig. 6. The lattice used in FDTD method.

The spatial and temporal points in the lattice are identified by means of whole index $m$ and $n$ as:

$$
X_{m}=m \Delta x ; \quad t_{n}=n \Delta t ;
$$

Based on the indices, current and voltage variable in space and time can be written as $I_{m}^{n}$ and $V_{m}^{n}$, as explained below.

The voltage spatial central finite difference formula is defined as:

$$
\frac{\partial}{\partial x} V(x, t) \cong \frac{V\left(x+\frac{\Delta x}{2}, t\right)-V\left(x-\frac{\Delta x}{2}, t\right)}{\Delta x}
$$

By using the notation with index $m$ and $n$, and taking into account that $\Delta \mathrm{x} / 2$ means half spatial step $(1 / 2)$, equation (8) can be rewritten as:

$$
\frac{\partial}{\partial x} V(m \Delta x, n \Delta t) \cong \frac{V_{m+1 / 2}^{n}-V_{m-1 / 2}^{n}}{\Delta x}
$$

Likewise, the current temporal central finite difference formula is given by: 


$$
\frac{\partial}{\partial t} I(x, t) \cong \frac{I\left(x, t+\frac{\Delta t}{2}\right)-I\left(x, t-\frac{\Delta t}{2}\right)}{\Delta t}
$$

As it was done for the voltage formula, by inserting the indices $m$ and $n$, equation (10) becomes:

$$
\frac{\partial}{\partial t} I(m \Delta x, n \Delta t) \cong \frac{I_{m}^{n+1 / 2}-I_{m}^{n-1 / 2}}{\Delta t}
$$

By replacing equations (9) and (11), the Telegraphist's' voltage equation (4), can be rewritten as:

$$
-\frac{V_{m+1 / 2}^{n}-V_{m-1 / 2}^{n}}{\Delta x}=R \cdot I_{m}^{n}+L \frac{I_{m}^{n+1 / 2}-I_{m}^{n-1 / 2}}{\Delta t}
$$

As it can be seen in the equation (12), the continue dependence of the voltage from the space $x$, described in equation (8), has been replaced by a discrete dependence from the index $m$. Furthermore, in equation (12), it is also possible to observe that the temporal index $n$ appears in the current expression is both entire $I_{m}^{n}$ and shifted by half quantity $I_{m}^{n \pm 1 / 2}$. In order to solve this equation, the average current at the instant $n$ has been introduced as:

$$
\begin{gathered}
-\frac{V_{m+1 / 2}^{n}-V_{m-1 / 2}^{n}}{\Delta x}= \\
R \cdot \frac{I_{m}^{n+1 / 2}+I_{m}^{n-1 / 2}}{2}+L \frac{I_{m}^{n+1 / 2}-I_{m}^{n-1 / 2}}{\Delta t}
\end{gathered}
$$

The FDTD technique is based on explicit formulas in which the term more advanced in time $I_{m}^{n+1 / 2}$ is written as a function of others quantities. Therefore, equation (13), after some mathematical manipulations, can be rewritten as:

$$
\begin{gathered}
I_{m}^{n+1 / 2}=I_{m}^{n-1 / 2}\left(\frac{2 L_{m}-R_{m} \Delta t}{2 L_{m}+R_{m} \Delta t}\right)+ \\
-\frac{2 \Delta t}{2 L_{m} \Delta x+R_{m} \Delta t \Delta x}\left(V_{m+1 / 2}^{n}-V_{m-1 / 2}^{n}\right)
\end{gathered}
$$

In the same manner, starting from the second Telegraphist's' equation describing the current behavior, the voltage term more advanced in time $\mathrm{V}_{\mathrm{m}}^{\mathrm{n}+1 / 2}$ can be obtained as follows:

$$
\begin{gathered}
V_{m}^{n+1 / 2}=V_{m}^{n-1 / 2}\left(\frac{2 C_{m}-G_{m} \Delta t}{2 C_{m}+G_{m} \Delta t}\right)+ \\
-\frac{2 \Delta t}{2 C_{m} \Delta x+G_{m} \Delta t \Delta x}\left(I_{m+1 / 2}^{n}-I_{m-1 / 2}^{n}\right)
\end{gathered}
$$

By observing equations (14) and (15), it is possible to notice that the FDTD technique allows to obtain a new information at time $n+1 / 2$ as a function of other information detected in the previous times $n$ and $n-1 / 2$ [23].

As it can be seen in equations (14) and (15), the voltage terms are those at time $n$ and its previous value $n-1 / 2$, respectively. Therefore, from equation (14) it is difficult to solve equation (15) and vice versa. This happens because the first one needs entire $n$ index, while the second equation needs half quantity of the $n$ index.

In the same way, for the current term which requires half $n$ index in equation (14) and entire $n$ index in equation (15). Therefore the two equations are not easily simultaneously solvable. Because it is possible to evaluate current and voltage in different spatial and temporal times, this problem can be overcome by adopting two lattices, one for the current and one for the voltage, different to that proposed in Figure 6. The double lattice is reported in Figure 7, in which current and voltage are evaluated in a given time and at spatial points, shifted by half quantity to respect to the previous lattice points.

In this figure the spatial and temporal points describing the voltage are highlighted in red color, while the points referred to the current are depicted in green color.

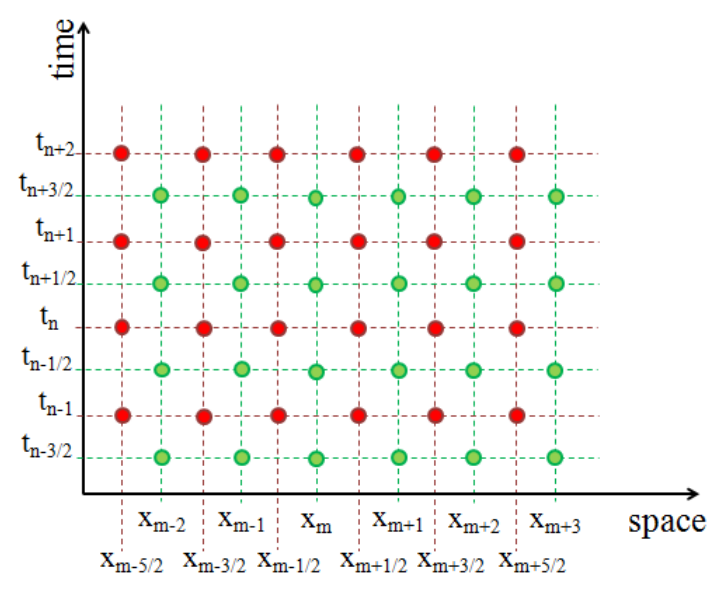

Fig. 7.The adopted double lattice.

By adding a half quantity $(+1 / 2)$ at each term, the equation (15) becomes:

$$
\begin{gathered}
V_{m+1 / 2}^{n+1}=V_{m+1 / 2}^{n}\left(\frac{2 C_{m+1 / 2}-G_{m+1 / 2} \Delta t}{2 C_{m+1 / 2}+G_{m+1 / 2} \Delta t}\right)+ \\
-\frac{2 \Delta t}{2 C_{m+1 / 2} \Delta x+G_{m+1 / 2} \Delta t \Delta x}\left(I_{m+1}^{n+1 / 2}-I_{m}^{n+1 / 2}\right)
\end{gathered}
$$

In this way, the current term in equations (16) can be calculated by using equation (14) and, vice versa, the voltage term in equation (14) can be evaluated by using equation (16) as:

$$
\begin{aligned}
I_{m}^{n+1 / 2} & =P_{m} \cdot I_{m}^{n-1 / 2}+Q_{m}\left(V_{m-1 / 2}^{n}-V_{m+1 / 2}^{n}\right) \\
V_{m+1 / 2}^{n+1} & =Y_{m+1 / 2} \cdot V_{m+1 / 2}^{n}+S_{m+1 / 2}\left(I_{m}^{n+1 / 2}-I_{m+1}^{n+1 / 2}\right)
\end{aligned}
$$

Where:

$$
\begin{gathered}
P_{m}=\frac{2 L_{m}-R_{m} \Delta t}{2 L_{m}+R_{m} \Delta t} ; \quad Q_{m}=\frac{2 \Delta t}{2 L_{m} \Delta x+R_{m} \Delta t \Delta x} ; \\
Y_{m+1 / 2}=\frac{2 C_{m+1 / 2}-G_{m+1 / 2} \Delta t}{2 C_{m+1 / 2}+G_{m+1 / 2} \Delta t} \\
S_{m+1 / 2}=\frac{2 \Delta t}{2 C_{m+1 / 2} \Delta x+G_{m+1 / 2} \Delta t \Delta x}
\end{gathered}
$$


This happens because, in both equations, all voltage terms are described by entire $n$ index and all current terms are described by half $n$ index.

However, equations (17) and (18) are implemented in order to describe the waves behavior in each PEA cell component, while, in correspondence of the nodes between two PEA cell components cascade connected, the continuity conditions of current and voltage waves are imposed by:

$$
\begin{gathered}
I L_{x}\left(M_{x}\right)=I L_{x+1}\left(m_{x+1}^{1}\right) \\
V L_{x}\left(M_{x}\right)=V L_{x+1}\left(m_{x+1}^{1}\right)
\end{gathered}
$$

with

$$
M=\sum_{i=1}^{n} m^{i}
$$

Where $m^{i}$ is the number of elements in which the PEA cell components, named $x$ and $x+1$, are described. The apex $i$ refers to the number of each element in witch is divided each component and $M$ is the sum of $m^{i}$ which corresponds to the last element of the related PEA cell component.

Therefore, the first term of equations (20) and (21) are referred to the current and voltage values assumed in the last spatial element of the generic component $x$, before the node. While, the second member is referred to the values assumed in the first spatial element of the next component $x+1$ (which is in contact with the component $x$ ), after the node. An example of components $x$ and $x+1$ and their spatial elements is depicted in Figure 8. In this way, the waves propagation behavior within the entire PEA cell is described.

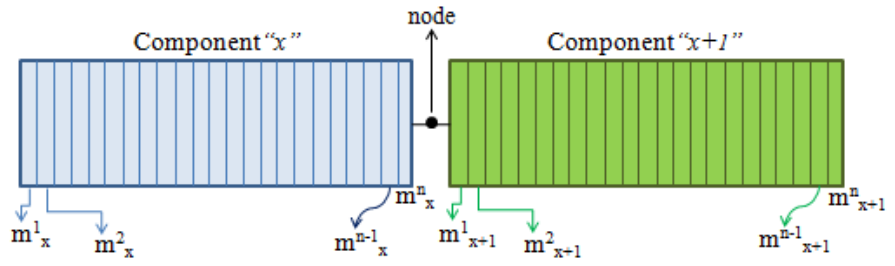

Fig. 8. Connection between two generic PEA cell components and related spatial elements.

Different boundary conditions are needed at the end of the transmission system, considering that when the acoustic waves reach the ends parts of the PEA cell (see Figure 4) are totally reflected. In this case an open-circuit has been adopted as boundary condition. For these reason, two resistances with high value $(\sim 1 \mathrm{M} \Omega)$ are inserted after the $\mathrm{HV}$ and bottom electrodes.

With reference to the piezoelectric sensor, instead, the Leach's impedance-type transducer model has been implemented. The complete explanation of the piezoelectric sensor model and the relative block diagram are well described in [24].

\section{THE PULSES GENERATOR}

As previously mentioned, the application of the pulse voltage $e_{p}(t)$ generates charges vibrations and thus the acoustic waves generation. The equation describing the acoustic waves generated from the surface charges $\sigma$, neglecting the charges induced by $e_{p}(t)$, is equal to [14]:

$$
p(t)=\sigma e_{p}(t)
$$

In accordance to the analogy between pressure and voltage, the acoustic waves generation can be modeled by means of voltage pulses sources $V_{p}$. In most cases, this waveform can be approximated by an ideal Gaussian pulse with magnitude $U$ depending on the external applied pulse voltage and width $\beta$ set around $9 \mu$ s. The equation describing this pulse source is given by:

$$
V_{p}=U e^{\frac{-(t-\alpha)^{2}}{\beta}}
$$

where the constant $\alpha$ is used to set the time delay.

The magnitude of $V_{p}$ is set in accordance to Equation (23), where $e_{p}(t)$ has been chosen equal to $600 \mathrm{~V}$. Furthermore, in a free space charge specimen of dielectric material the same quantity of interfacial charges is present in both dielectric interfaces, thus the same value of magnitude $U$ is used for the two pulse generators. In particular, the pulse generators (with polarity depending on the sign of the accumulated interfacial charges) are placed in the same points in which the charges are accumulated. For this reason, in order to simulate the acoustic waves generation from the specimen interfaces, two pulse sources have been inserted in series to the transmission line representing the specimen, as shown in Figure 9.

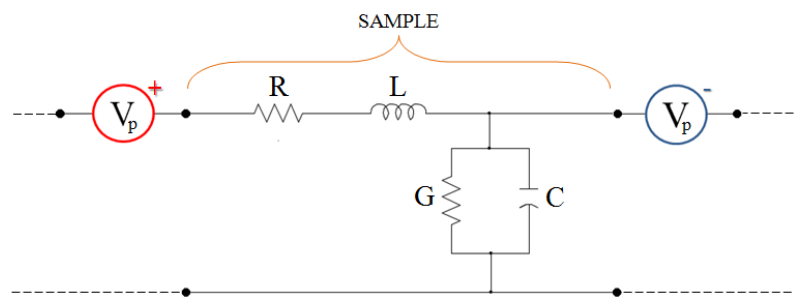

Fig. 9. Schematic representation of the pulse generators inserted in correspondence of the transmission line representing the specimen. The red and blue colors are used to differentiate the positive and the negative polarity, based on the interfacial accumulated charge sign.

\section{EXPERIMENTAL VALIDATION OF THE DEVELOPED MODEL VI-I. SIMULATION RESULTS}

The possibility of recognizing and distinguishing the reflections from the real signal of the accumulated charge is one of the main purposes of the model. With this aim, a 200 $\mu \mathrm{m}$ thickness LDPE single layer sample has been used according to the parameters proposed in [14]. The DC input voltage of $10 \mathrm{kV}$ is applied in order to have an electric field stress of $50 \mathrm{kV} / \mathrm{mm}$ across the LDPE sample, considering a dielectric permittivity $\varepsilon_{r}=2.3$. These conditions determine 1 
$\mathrm{mC} / \mathrm{m}^{2}$ positive and negative accumulated charge in both interfaces of the PE sample. Furthermore, as explained in section $\mathrm{V}$, the pulse sources, implemented in the model in order to be proportional to the accumulated charge, are set to $600 \mathrm{~V}$ for both positive and negative charges.

The simulated output signal, obtained by using the same dimensional characteristics listed in Table II of [14], is shown in Figure 10.

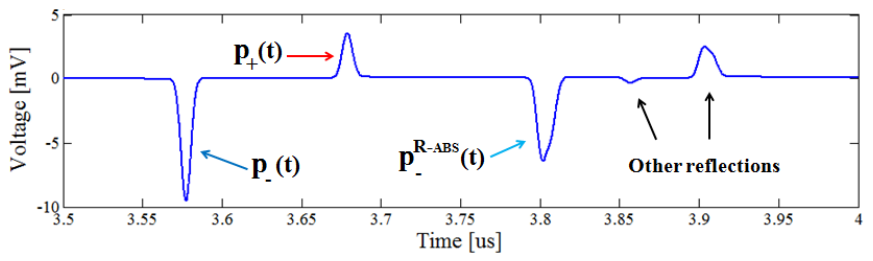

Fig. 10. Space charge profile output signal of the PEA cell model received by the transducer without reflections.

The space charge profile, thanks to the good agreement between the sample features and the PEA cell components, shows the positive $\left(p_{+}(t)\right)$ and the negative $\left(p_{-}(t)\right)$ signals not affected by reflections. The transducer receives the first reflection, due to the absorber, after around $3.8 \mu \mathrm{s}$.

Otherwise, if the sample thickness is greater than a certain value, as demonstrated in Section 3, the PEA cell main output signal can be affected by reflections. In fact, if the maximum sample thickness $d_{S a}$ not satisfy the inequality of Equation (1), the main peak, $p_{+}(t)$, referred to the positive surface charge, will be preceded by the reflected wave within the ground electrode, $p_{-}^{R_{G}}(t)$.

As a confirmation of the theoretical validation of the model, another two examples, similar to that proposed in [14], are carried out below.

In the first one, the effect of the ground electrode thickness $d_{G R}$ is analyzed. In particular, after simply manipulation of Equation (1) and considering the same dielectric sample used in the previous simulation with $v_{L D P E}=1950 \mathrm{~m} / \mathrm{s}, d_{G R}$ should be greater than $330 \mu \mathrm{m}$. Thus, considering the same single layer configuration of Figure 2, if $d_{G R}$ is equal to $325 \mu \mathrm{m}$, $p_{-}(t)$ reaches the transducer after $\tau_{G R}=0.05 \mu$ s and $p_{+}(t)$ after $\tau_{S a}+\tau_{G R}=0.153 \mu \mathrm{s}$, while $p_{-}^{R_{G}}(t)$ after $3 \tau_{G R}=0.150$ $\mu \mathrm{s}$. With these values, the reflected signal $p_{-}^{R_{G}}(t)$ appears in the space charge profile superimposed to $p_{+}(t)$, as shown in Figure 11.

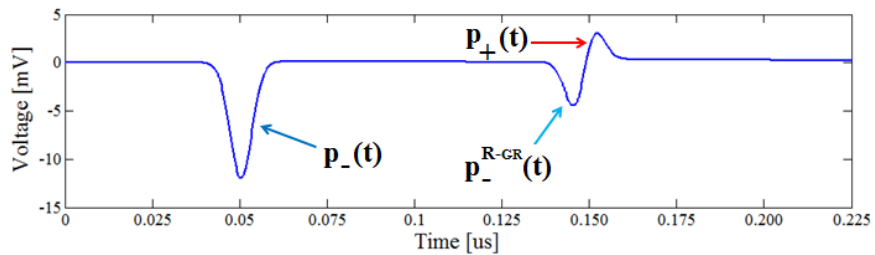

Fig. 11. Output of the PEA cell. Signal distortion due to the reflection within the ground electrode

In the second example, instead, the effect of the absorber thickness $d_{A B S}$ is analyzed. In this case, referring to the configuration scheme of Figure 3 and manipulating Equation (2), the absorber thickness $d_{A B S}$ must be greater than $116 \mu \mathrm{m}$ in order to avoid that the reflection $p_{-}^{R_{A B S}}(t)$ reaches the transducer before than the signal $p_{+}(t)$. Indeed, for a $20 \mathrm{~mm}$ thick ground electrode, if $d_{A B S}$ is chosen $100 \mu \mathrm{m}$, $p_{-}^{R_{A B S}}(t)$ reaches the transducer after $3.67 \mu$ s, while $p_{+}(t)$ and $p_{-}(t)$ reach the transducer after $3.68 \mu \mathrm{s}$ and $3.58 \mu \mathrm{s}$, respectively. Therefore, the false signal $p_{-}^{R} A B S(t)$ occurs in the main output signal, superimposed to $p_{+}(t)$, as shown in Figure 12.

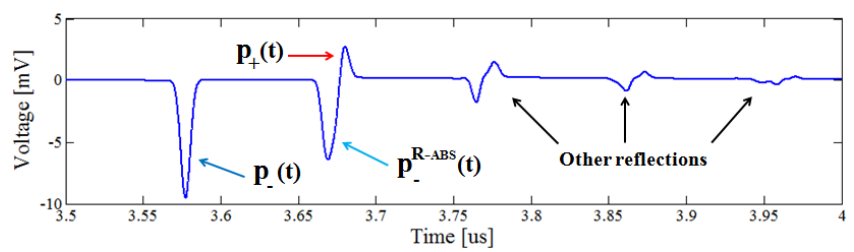

Fig. 12. Output of the PEA cell. Signal distortion due to the reflection within the absorber.

\section{VI-II. EXPERIMENTAL RESULTS}

In order to experimentally validate the developed PEA cell model, experimental measures have been carried out by means of two PEA cells. Firstly, the PEA cell of the TDHVL (with ground electrode thickness $2 \mathrm{~mm}$ and absorber thickness 450 $\mu \mathrm{m})$ has been adopted. The specimen under test is a $100 \mu \mathrm{m}$ thick LDPE foil with attenuation coefficient $\alpha$ equal to 4873 $\mathrm{Np} / \mathrm{m}$ and speed of sound $\mathrm{v}=2200 \mathrm{~m} / \mathrm{s}$. After 2 minutes of applied voltage (useful to observe only surface charges) the obtained charge distribution is shown in Figure 13.



Fig. 13. PEA cell output signal obtained by experimental test, for LDPE 100 $\mu \mathrm{m}$ thick.

Considering that both Equations (1) and (2) are satisfied, no reflected waves are present in the main PEA cell output signal. By implementing the PEA cell of the TDHVL and the specimen under test into the model, the simulation result is reported in Figure 14. In this case, for a better comparison between experimental and simulation results, the output signal obtained by the model has been amplified and calibrated.



Fig. 14. PEA cell output signal obtained by simulation test, for LDPE $100 \mu \mathrm{m}$ thick.

The signal amplification has been made by inserting a gain block (with the same value of the amplifier used in the real PEA cell) after the sensor, while the signal calibration (useful 
for the conversion from $\mathrm{mV}$ to $\mathrm{C} / \mathrm{m}^{3}$ ) has been made by applying the technique described in [25].

As can be seen, the obtained PEA cell output signals in both experimental (figure 13) and simulation (Figure 14) test, are very similar to each other. Therefore, the experimental validation of the model is obtained.

In order to confirm the performance of the developed model, another PEA cell with different features has been adopted. The second PEA cell is that of the LEPRE lab, and in this case the specimen under test has been selected with the aim to provide a wave reflection in the main output signal of the PEA cell. This PEA cell has an absorber layer with thickness $250 \mu \mathrm{m}$ and sound velocity $2260 \mathrm{~m} / \mathrm{s}$. Therefore, by using a dielectric layer $0.69 \mathrm{~mm}$ thick with sound velocity $1189 \mathrm{~m} / \mathrm{s}$, the Equation (2) is not fulfilled. Figure 15 shows the PEA cell output signal depicted by the scope, as can be seen a reflected signal $p_{-}^{R_{A B S}}(t)$ is present between the two main peaks.

Considering the presence of a reflection, the deconvolution software (useful to compensate the high pass filter effect due to the transducer-amplifier connection) could not be used. This because it works well when a zero signal is present between the two main peaks [25]. Therefore, also the calibration procedure (which it is typically made after the signal deconvolution) has not been applied.

In Figure 16, instead, simulation result is shown. In both simulation and experimental results, due to the sample and PEA cell features, a negative peak falls in the middle of the main PEA output signal. As can be seen, this reflected peak could be easily confused as an accumulation of space charges in the insulation bulk if the acoustic wave behavior within the PEA cell is unknown. From this, the importance of the proposed PEA cell model which can be used to avoid incorrect interpretation of the real accumulated space charge.

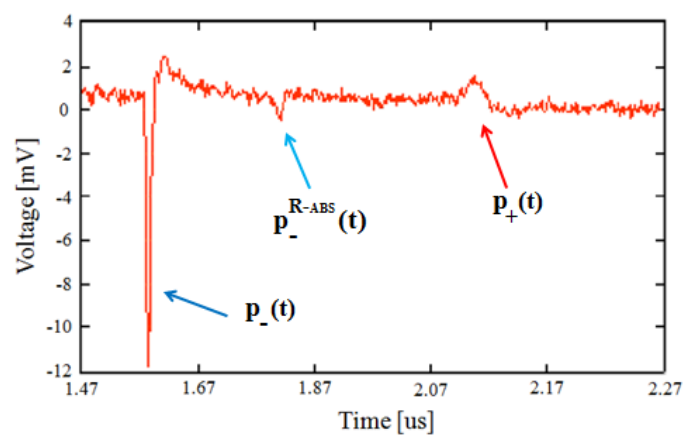

Fig. 15. Experimental PEA cell output signal provided by the scope. Due to the sample and absorber features, a reflected peak is present in the middle of the main output signal.

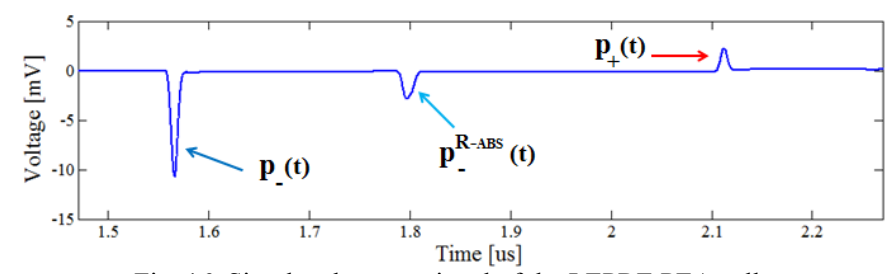

Fig. 16. Simulated output signal of the LEPRE PEA cell.

\section{ACCUMULATED SPACE CHARGE}

To reach the sensor, the acoustic waves, generated by the space charges deposited within the sample, need a lower time, compared to that needed for the acoustic waves generated by the surface charges accumulated in the HV-electrode/sample interface. This means that, if the Equations (1) and (2), referred to a specimen with only surface charges are satisfied, even with accumulated space charge, no reflected signals appear in the main PEA output signal.

Beyond this, in this paragraph the main objective is to demonstrate that the developed model is able to simulate the PEA output signal even when an amount of space charge is accumulated within the sample.

In order to obtain this, the PEA cell of the LEPRE lab has been used, and the experimental space charge profile, obtained by stressing for 1 hour an XLPE specimen $0.39 \mathrm{~mm}$ thick, is reported in Figure 17.

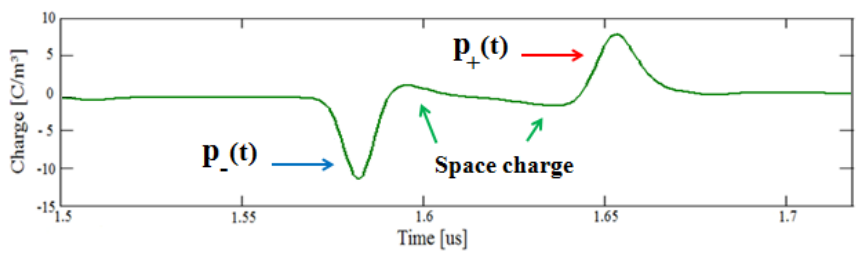

Fig. 17. Experimental profile in presence of accumulated space charges.

Starting from the charge profile of Figure 17, the developed model is able to read the charge quantities distributed within the sample and to insert the pulse generators according to the generated pressure waves $\mathrm{p}_{\rho}(\mathrm{t})$ described by Equation (25).

$$
p_{\rho}(t)=\rho\left(\lambda_{k}\right) \Delta \lambda e_{p}(t)
$$

Where $\rho$ is the amount of space charge in the position $\lambda_{\mathrm{k}}, \Delta \lambda$ is the thin slice of the sample in which the charges are accumulated, while $e_{p}(t)$ is equal to $600 \mathrm{~V}$ (the same value of that applied experimentally). Because the experimental PEA cell acquisition software provides a measured charge vector of 196 units, the simulated sample has been divided in 196 parts as well. Therefore 196 pulse generators has been inserted within the sample, one of each $\Delta \lambda=0.002 \mathrm{~mm}$.

As can be seen in the simulated profile reported in Figure 18, the magnitude of $\mathrm{p}_{+}(\mathrm{t})$ is a bit smaller compared to that obtained experimentally in Figure 17. This is probably due to the small difference between the calculated and the real attenuation factor of the dielectric material.

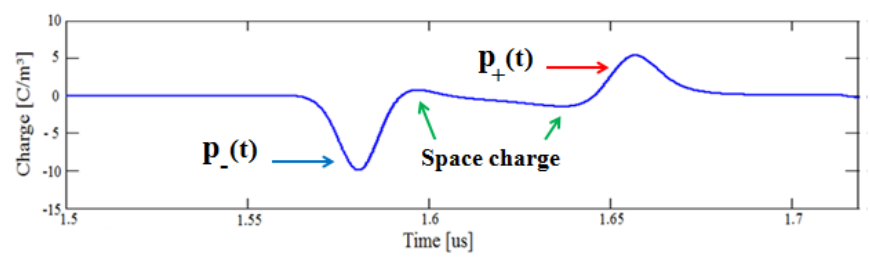

Fig. 18. Simulated profile in presence of accumulated space charges.

Another difference consists in the width of the peaks of the 
simulated profile. This because in the elaboration software of the LEPRE PEA cell, the signal is recovered by taking into account the dispersion factor. In the developed model, instead, it has not been considered, but certainly it will be an objective for future works, which can be made by following the technique described in [26]. A further interesting software development could be the implementation of the charge estimation equations [27-28], with the aim to realize a complete charge profile simulation model.

\section{CONCLUSION}

The purpose of this work was to develop a model able to simulate the acoustic wave behavior within the PEA cell. The proposed work highlights the effect of the PEA cell component and sample features on the PEA output signal. In particular, it was found that, for a specific PEA cell, the sample features should be carefully chosen in order to avoid distortion of the PEA output signal. This means that for a given PEA cell, the thickness of the dielectric materials that can be tested it is strongly related with his specific physical properties as the sound speed. This has been demonstrated by simulations and confirmed by experimental test. In particular, in both simulation and experimental charge distribution obtained with the PEA cell of the LEPRE lab, because the material properties of the dielectric layer fall outside the range allowed by the PEA cell, a reflection occurs. This reflection, due to the absorber features, which is finally located within the main PEA output signal, could be confused as an accumulation of space charge and thus the output profile may be misinterpreted.

The model validation was also made by comparing the simulation results with those proposed in literature. In addition, by using the PEA cell of the TDHVL lab, the comparison between simulation and experimental charge profiles confirmed the good performance of the developed PEA cell model.

Moreover, a simulation of the PEA output profile has been made in case of accumulated space charges within the sample. Although there are some small differences, also in this case the model shows a good agreement with the charge distribution carried out experimentally.

Based on the reached results, different evolutions of the model are possible in future works. As for example, the implementation of the charge estimation equations and simulation of the acoustic wave behavior in case of multilayer specimens.

\section{REFERENCES}

[1] L. A. Renforth, R. Giussani, M. T. Mendiola and L. Dodd, "Online Partial Discharge Insulation Condition Monitoring of Complete High-Voltage Networks," in IEEE Transactions on Industry Applications, vol. 55, no. 1, pp. 1021-1029, Jan.-Feb. 2019.

[2] L. A. Renforth, R. Armstrong, D. Clark, S. Goodfellow and P. S. Hamer, "High-Voltage Rotating Machines: A New Technique for Remote Partial Discharge Monitoring of the Stator Insulation Condition," in IEEE
Industry Applications Magazine, vol. 20, no. 6, pp. 79-89, Nov.-Dec. 2014.

[3] B. Yazici, "Statistical pattern analysis of partial discharge measurements for quality assessment of insulation systems in high-voltage electrical machinery," in IEEE Transactions on Industry Applications, vol. 40, no. 6, pp. 1579-1594, Nov.-Dec. 2004.

[4] L. A. Renforth, P. S. Hamer, D. Clark, S. Goodfellow and R. Tower, "Continuous Remote Online Partial Discharge Monitoring of HV Ex/ATEX Motors in the Oil and Gas Industry," in IEEE Transactions on Industry Applications, vol. 51, no. 2, pp. 1326-1332, March-April 2015.

[5] J. E. Timperley and J. M. Vallejo, "Condition Assessment of Electrical Apparatus With EMI Diagnostics," in IEEE Transactions on Industry Applications, vol. 53, no. 1, pp. 693-699, Jan.-Feb. 2017.

[6] T. Takada, "Space charge formation in dielectrics," IEEE Trans. Elect. Insul., vol. EI-21, no. 6, pp. 873-879, Dec. 1986.

[7] L. A. Dissado and J. C. Fothergill, "Electrical Degradation and Breakdown in Polymers", P. Peregrinus Press, London,U. K., 1992.

[8] J. C. Fothergill and L. A. Dissado, "Space charge in solid dielectrics," Dielectrics Society, Charity U.K, 1998.

[9] G. Mazzanti, M. Marzinotto, "Space Charge in HVDC extruded insulation: storage, effect, and measurement methods", in Extruded Cables for High Voltage Direct Current Transmission: Advances in Research and Development, Power Engineering Series, Wiley-IEEE Press, New York, USA, 2013, pp. 99-207.

[10]Y. Li, M. Yasuda, T. Takada,"Pulsed electroacoustic method for measurement of charge accumulation in solid dielectrics," Dielectrics and Electrical Insulation, IEEE Transactions on, vol.1, No.2, pp.188-195, Apr 1994.

[11]G. Ala, M. Caruso, V. Cecconi, S. Ganci, A. Imburgia, R. Miceli, P. Romano and F. Viola, "Review of acoustic methods for space charge measurement," 2015 AEIT International Annual Conference (AEIT), Naples, Italy, 2015.

[12]R. Bodega, P. H. F. Morshuis and J. J. Smit, "Electrostatic force distribution in a multi-layer dielectric tested by means of the PEA method," Proceedings of the 2004 IEEE International Conference on Solid Dielectrics, 2004. ICSD 2004., 2004, pp. 264-267 Vol.1.

[13] Mohamad Abed A. LRahman Arnaout (2013), "Acoustic Wave Propagation in a Pulsed Electro Acoustic Cell", Wave Propagation Theories and Applications, Dr. Yi Zheng (Ed.), INTECH pp.25-42, 2013.

[14]J. S. Chahal and C. C. Reddy, "Modeling and Simulation of Pulsed Electroacoustic Measurement Method," in IEEE Systems Journal, vol. 8, no. 4, pp. 1283-1292, Dec. 2014.

[15]A. Imburgia, R. Miceli, E. R. Sanseverino, P. Romano and F. Viola, "Review of space charge measurement systems: acoustic, thermal and optical methods," in IEEE Transactions on Dielectrics and Electrical Insulation, vol. 23, no. 5, pp. 3126-3142, October 2016.

[16]P. Romano, A. Imburgia, "Effect of Acoustic Wave Reflections on Space Charge Measurements with PEA Method" IEEE 4th International Forum on Research and Technology for Society and Industry (RTSI), Palermo, Italy, pp. 1-6, Sept. 2018.

[17] K. Kumaoka, T. Kato, H. Miyake and Y. Tanaka, "Development of space charge measurement system with high positionalal resolution using pulsed electro acoustic method," Proceedings of 2014 International Symposium on Electrical Insulating Materials, Niigata, 2014, pp. 389392.

[18]R. Bodega, P. H. F. Morshuis and J. J. Smit, "Space charge measurements on multi-dielectrics by means of the pulsed electroacoustic method," in IEEE Transactions on Dielectrics and Electrical Insulation, vol. 13, no. 2, pp. 272-281, April 2006.

[19]C. Tang, B. Huang, M. Hao, Z. Xu, J. Hao, G. Chen, "Progress of Space Charge Research on Oil-Paper Insulation Using Pulsed Electroacoustic Techniques", Energies, vol. 9, pp. 53, 2016.

[20]B. Bauer, "Equivalent circuit analysis of mechano. Acoustic structures," in Transactions of the IRE Professional Group on Audio, vol. AU-2, no. 4, pp. 112-120, July-August 1954.

[21] J. van Deventer, T. Lofqvist, and J. Delsing, "PSpice simulation of ultrasonic systems," IEEE Trans. Ultrason., Ferroelectr., Freq. Control, vol. 47, no. 4, pp. 1014-1024, Jul. 2000.

[22]F. Viola, P. Romano and R. Miceli, "Finite-Difference Time-Domain Simulation of Towers Cascade Under Lightning Surge Conditions," in 
IEEE Transactions on Industry Applications, vol. 51, no. 6, pp. 49174923, Nov.-Dec. 2015.

[23]A. Taflove, S. Hagness, "Computational Electrodynamics: The FiniteDifference Time-Domain Method", Artech House, Boston, MA, 2000.

[24] W. Marshall Leach, Jr., "Controlled-source analogous and SPICE model for piezoelectric transducers," IEEE Trans. Ultrason., Ferroelectr., Freq. Control, vol. 41, no. 1, pp. 60-66, Jan. 1994.

[25] M. J.P. Jeroense, "Charge and discharges in HVDC cables. In particular in mass-impregnated HVDC cables", Delft University Press, the Netherlands 1997

[26] Y. Li, M. Aihara, K. Murata, Y. Tanaka, and T. Takada, "Space charge measurement in thick dielectric materials by pulsed electroacoustic method”, Rev. Sci. Instrum., Vol. 66, No. 7, July 1995.

[27]G. Mazzanti, G. C. Montanari and J. M. Alison, "A space-charge based method for the estimation of apparent mobility and trap depth as markers for insulation degradation-theoretical basis and experimental validation," in IEEE Transactions on Dielectrics and Electrical Insulation, vol. 10, no. 2, pp. 187-197, April 2003.

[28]K. Kaneko, T. Mizutani and Y. Suzuoki, "Computer simulation on formation of space charge packets in XLPE films," in IEEE Transactions on Dielectrics and Electrical Insulation, vol. 6, no. 2, pp. 152-158, Apr 1999.

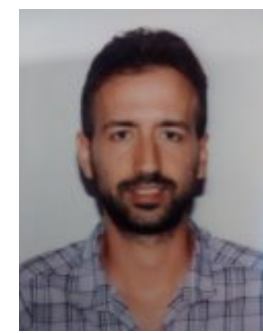

Antonino Imburgia was born in Palermo, Italy, on 28 April 1987. He received the M.Sc. degree in electrical engineering from the University of Palermo in 2014. From 2015 he is a Ph.D. student at the Department of energy, information engineering and mathematical models (DEIM) at the University of Palermo. His current research is in the field of HVDC, distribution of space charge in solid dielectrics and its behavior, dielectrics and electrical insulating systems diagnosis, space charge and partial discharge measurements.

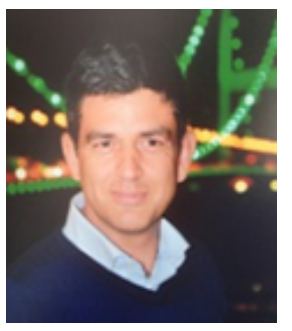

Pietro Romano (SM'17) was born in Palermo, Italy on 15 May 1967. He received the MSc. and $\mathrm{Ph} . \mathrm{D}$. degrees in electrical engineering from the University of Palermo, Italy, in 1993 and 1998, respectively. From 1998 to 2001, he worked at CRES Centre of Electronic Research on Sicily as researcher on partial discharge measurements. From 2001 he is a researcher at the Department of Electrical Engineering at the University of Palermo and teaches Basic Electrical Engineering, Electrotechnics and insulating materials. His research activity is mainly in the field of insulating systems diagnosis, partial discharge measurements, HV Systems, multifactor stress effects and electric field simulations, partial shaded PV Systems.

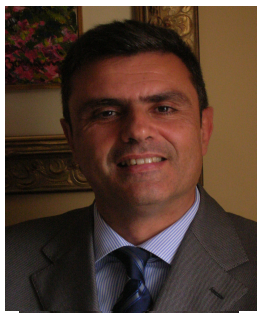

Prof. Dr. Guido Ala (SM'16) was born in 1964. In 1989 he earned the "Laurea" degree (MD) in Electrical Engineering from the University of Palermo, Italy. In 1994 he earned the Ph.D. degree in Electrical Engineering from the Italian MIUR. From 1992 to 1996 he was a teacher (permanent staff) of Electrothecnics at the Italian technical high schools. In 1997 he worked as an energy engineer in the Municipal Gas Company in Palermo. Then he joined the University of
Palermo, where he was appointed as a researcher from 1997 to 2004. Since 2005 he has been an associate professor, now at the Department of Engineering. In 2017 he attained the National Scientific Qualification for the role of full professor in Electrical Engineering. His main research interests are in the fields of computational electromagnetics, grounding systems, lightning, electromagnetic compatibility, partial discharge detection, bio-electromagnetic numerical modeling, electrical analogies in viscoelasticity. He serves as a reviewer for several International Journal. He is Associate Editor of the International Journal of Numerical Modelling: Electronic Networks, Devices and Fields- Wiley. For the same Journal he was Guest Editor of a Special Issue on Advances in ElectroMagnetic Compatibility (EMC) numerical modelling, in 2018. In 2013, he has been among the (ten) Distinguished Reviewers of IEEE Transactions on EMC. He is member of the Advisory Committee in 19th IEEE Mediterranean Electrothecnical Conference, May 2-7, 2018, Marrakesh, Marocco. He is Local co-Chair in 18th IEEE International Conference on Environment and Electrical Engineering (IEEE EEEIC18) e 2nd IEEE Industrial and Commercial Power System Conference EUrope (I\&CPS Europe), June 12-15, 2018, Palermo, Italy. He is General co-Chair in 4th IEEE International Forum on research and Technologies for Society and Industry (IEEE RTSI 2018), September 10-13, 2018, Palermo, Italy. $\mathrm{He}$ is General co-Chair in 20th IEEE Mediterranean Electrothecnical Conference (IEEE MELECON 2020), June 16-18, 2020, Palermo, Italy.

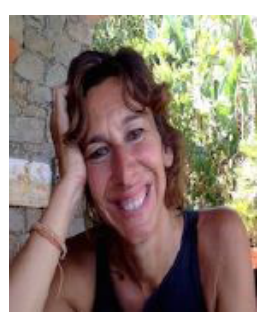

Eleonora Riva Sanseverino In 1995 she took the degree in electrical engineering at the Faculty of engineering at the University of Palermo. In 2001 she was researcher at the Computer Science in the National Council of Research, Institute ICAR of Palermo. In 2002 she got a position of Associate Professor SSD INGIND/33 (Power Systems) at the University of Palermo. Since 2013 she is a Full Professor in Power systems by the Italian Ministry of Education. She authored more than 140 scientific contribution on international journals and proceedings of international conferences and 2 manuscripts edited by Springer and Francoangeli.



Graziella Giglia received the degree in Electronic Engineering and Infotelematic Applications with honors in 2006 and the Master's degree in Electronic Engineering with honors in 2010 from the University of Palermo. In 2017 she earned the Ph.D. degree in Energy and Information Technologies at the University of Palermo, and in collaboration with the CNR Institute of Intelligent Systems for Automation (ISSIA). Her main research interests are electrical drive systems, electromagnetic interference (EMI) reduction, electromagnetic compatibility, grounding systems, lightning, partial discharge detection, and development and implementation of control algorithms on FPGA and DSP microprocessor platforms. 